\title{
High-protein diet fends off hunger
}

A high-protein diet increases the secretion of a hunger-curbing hormone, leading to reduced calorie intake and weight loss in mice. These results may explain why highprotein diets, such as Atkins, are so effective in helping people lose weight.

The composition of the typical Western diet includes about half as much protein as the diet thought to have been consumed by our hunter-gatherer ancestors, and this deficiency may, at least in part, be responsible for the skyrocketing rates of obesity seen in developed nations. Several studies have demonstrated that high-protein diets promote satiety and lead to weight loss in people, but the basis of this effect has remained unclear.

Now, Rachel L. Batterham of University College London (London, UK) and colleagues report in the September issue of Cell Metabolism that the anorectic hormone peptide YY (PYY) may play a key role in suppressing hunger. PYY is one of several hormones responsible for regulating energy homeostasis; it is released in the gastrointestinal tract in response to a meal and sends signals to the brain indicating satiety. Batterham's team found increased levels of the hormone in people following a high-protein meal, compared to high-fat or high-carbohydrate meals containing the same number of calories.

To further investigate the relationship between PYY and a meal's protein content, the researchers turned to a mouse model. They found that mice fed a highprotein diet consumed fewer calories and showed increased levels of PYY. Chronic consumption of high-protein chow led to decreased weight gain and reduced deposition of adipose tissue, as well as increased levels of PYY.

Next, to confirm the role of PYY in regulating calorie intake, the researchers created a line of PYY knockout mice. The mice that could not produce PYY ate more than wildtype mice and became obese, even when fed a high-protein diet. Chronic administration of PYY, however, resulted in dramatic weight loss-approximately $20 \%$ of body weight after 15 days of daily injections.

Protein's increased ability to reduce feelings of hunger as compared to carbohydrates or fat may have an evolutionary basis. As the authors write in Cell Metabolism, "Many of the physiological systems that

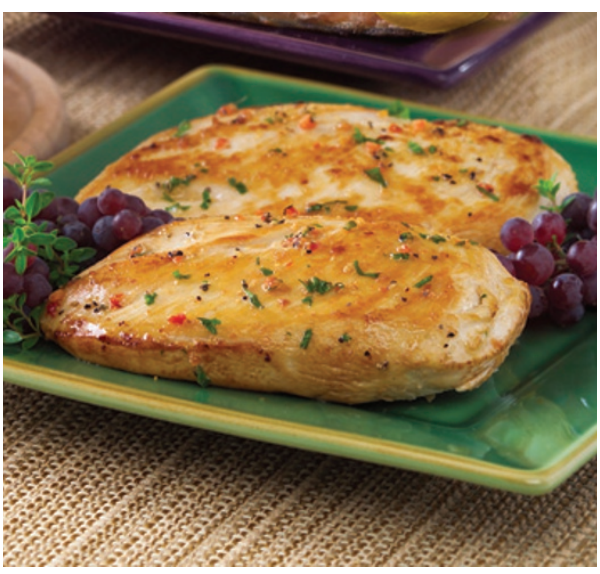

regulate food intake were probably established and may function better under lower-carbohydrate and higher-protein dietary conditions."

These results suggest that boosting your diet's protein content may be a viable weight-loss strategy. It should be noted, however, that large-scale clinical trials have yet to confirm this idea.

Tanja Schub

\section{ICTLIN PROVES A POWEREUI_SOOTHER OF PAIN}

Recent research into the neurological pathways of temperature sensation portends relief for those suffering from chronic pain. The new study, published in the 22 August issue of Current Biology, investigates the mechanism by which menthol and icilin, two compounds known to create 'sensations of coldness' in rats and humans, induce analgesia in rats.

The analgesic effects of cold and menthol have been recognized for many centuries, but the neurological mechanism that mediates that effect has been unknown. Researchers at the University of Edinburgh (Scotland) set out to test whether TRPM8, a 'coolsensitive' transient receptor potential cation channel, is involved in the pain-relief mediation of molecules that create coldness sensations, such as menthol and icilin. As an experimental model, Susan Fleetwood-Walker, Rory Mitchell, and their colleagues chose adult male Wistar rats with chronic constriction injury to sciatic nerve; these animals are reflexively sensitive to certain stimuli, the degree of which can be measured by their behavior.

To test TRPM8's involvement in the analgesic effects of coldmimicking molecules, Fleetwood-Walker and Mitchell applied solutions of menthol or icilin (either topically on the affected paw or intrathecally) to the rats and measured their sensitization to thermal and mechanical stimuli as compared to untreated controls. Low concentrations of both solutions of menthol and icilin significantly reversed the sensitization of the rats, though icilin had a more marked effect. In a subsequent experiment, the researchers 'knocked down' TRPM8 levels in some of the rats using antisense oligonucleotides; they then repeated their original experiment on these 'knockdown' rodents in order to determine whether the sensitization reversal involved TRPM8. They discovered that the knockdown nullified the reversal effects of icilin, thereby incriminating TRPM8 as a vital player in cold-induced analgesia.

Fleetwood-Walker and Mitchell hope these positive results will soon translate into chronic pain treatment for humans, especially a treatment for neuropathic pain. "Neuropathic pain is inadequately treated using current analgesic drugs," the authors tell Lab Animal, "Clinically, we expect that effective pain relief, with little or no side effects, will be achieved by topical application of TRPM8 activators in a cream or similar vehicle." But the work is far from finished: "Much more needs to be done," the authors conclude, "to fully clarify the molecular mechanisms involved and thereby potentially generate further new targets for therapeutic intervention." Owen Young 